\title{
TEST AUTOMATION FOR NEW GENERATION XAML WEB APPLICATIONS USING MULTI AGENT SYSTEM
}

\author{
Appasami G. ${ }^{1}$, Suresh Joseph $\mathrm{K}^{2}$, Balaji Srikaanth $\mathbf{P}^{3}$ \\ 1,3 Department of CSE, Dr. Pauls Engineering College, Villupuram, Tamilnadu, India, \\ Affiliated to Anna University Chennai \\ ${ }^{2}$ Department of computer science, Pondicherry University, Pondicherry, India, \\ Email: 1appas_9g@yahoo.com, 22 sureshjosephk@yahoo.co.in, ${ }^{3}$ srikaanth@yahoo.com
}

\begin{abstract}
New generation of Interactive and attractive dynamic web applications are developed by Silverlight Technology. It is developed by Microsoft on Dot Net 3.5 Frame work using eXtended Application Markup Language (XAML). User Interface Test Automation for Silverlight Applications plays a vital role in software industry; especially User Interface Test Automation (UITA) in new technology like Silverlight is a challenging task because of its high security and low accessibility. Agent Technology is intermediate software that provides a better bridge between User Interface Test Automation and Silverlight applications. There are many different software agents for each group of controls to do User Interface Test Automation. For example button control agent will take care of all buttons in User Interface Test Automation. In this paper we present the XAML User Interface test automation using Multi Agent System. The main goal of Test Automation for New Generation XAML Web Applications Using Multi Agent System are to reduce cost and time to perform User Interface Test Automation.
\end{abstract}

Key words: - User Interface, Test Automation, Silverlight XAML Applications and Multi Agent system Test Automation.

\section{INTRODUCTION}

Today world is in need of sophisticated interactive and attractive web applications for users. Silverlight is one of the new technologies to provide such web controls with maximum security. Silverlight applications are used to create platform independent, browser independent interactive and attractive web applications with Dot net supporting languages. Silverlight is Microsoft's new cross browser or delivering richer interactive applications to users over the web. Silverlight 2.0 is Microsoft's second release of the Silverlight platform [18][19]. Silverlight 2's biggest change from Silverlight 1.0 is the inclusion of a compact version of the .NET Framework, complete with the .NET Framework 3.0 Common Language Runtime. By adding .NET to Silverlight, Microsoft makes it easy for .NET developers to reuse their existing programming skills, collaborate with designers, and quickly create rich applications for the Web. One of the key benefits of Silverlight 2 is that it can execute any .NET language, including C\# and VB.NET [17][18].

Silverlight 2 ships with a "lightweight" version of the full .NET Framework, which features, among other classes, extensible controls, XML Web Services, networking components, and LINQ APIs. This class library is a subset of the .NET Framework's Base Class
Library, which enables the Silverlight plug-in to be a fast and small download. In addition to the .NET Framework classes, Silverlight 2 also ships with a subset of the WPF UI programming model, including support for shapes, documents, media, and WPF animation objects [15][18][19].

Agents are very useful to perform particular task by observing the environment and to react on environment. Agents will act as domain experts in $\mathrm{UI}$ Test Automation. To reduce the work load of Test Automation we are going to use Multi Agent System (MAS).Each agent will observe the environment and react on the environment by perception. Each Agent plays a vital role in UI Test Automation. For example text box agent will take care of all text boxes. It will act as an inter mediator for UI Test Automation and Silverlight applications. Agents will decide themselves to satisfy their design objectives. Agents are best in splitting record values and send particular values to particular agents. Each agent will take care of group of similar controls. So we can say agents are Inter mediator in UI Test Automation of Silverlight applications. 
Appasami et al : Test Automation for New Generation XAML Web Applications...

\section{SILVERLIGHT}

Silverlight means eXtented Application Markup Language (XAML). It is a Graphical User Interface Design Language (GUIDL). Silverlight is Microsoft's new cross browser or delivering richer interactive applications to users over the web. Silverlight 2.0 is Microsoft's second release of the Silverlight platform. Silverlight is a Microsoft .NET new UI Language for attractive web applications. Silverlight is developed my Microsoft on Dot Net 3.0 Frame work. So Silverlight provides security up to the level of Dot Net 3.0 Frame work. But Silverlight controls are written by a language called XAML. Silverlight 2 ships with a "lightweight" version of the full .NET Framework, which features, among other classes, extensible controls, XML Web Services, networking components, and LINQ APIs.

\section{Advantages of Silverlight are:}

* Micro Soft new technology for web

* Extension of ASP.NET

* Improved visualization

* XAML language for UI description

- Rich Interactive \& Attractive Web Applications

* work in all platforms and in all browsers

* Animated Movies can be sent to client with compressed data

* Rich Multimedia, Audio, Video \& Animation Support

* Developing device Independent components

* Combined work of Flash and .Net

* write-once-run-everywhere

* WPF-subset and .NET-subset

\section{USER INTERFACE TEST AUTOMATION}

Testing of $\mathrm{UI}$ is always challenging and has been mostly manual till now. In the next generation of MES applications the testing of GUI needs to be automated as it will save lot of man-hours that is lost in manual testing also it will catch the defects early on in the cycle.

$\mathrm{UI}$ Test Automation is a combined process of UI Testing and UI Automation. The data flow of UI Test Automation is shown in figure 1. Usability Testing is a mode of testing a particular product for its compatibility in terms of use. GUI testing is a commonly known form of Usability Testing of software or website. It is also known as User Interface Testing. GUI testing is a performance related assessment of a software or website in terms of ease of use, versatility, friendliness with focus on the target audience, visual impact and the approach and time taken to progress into the specific purpose.

UI Test Automation is a process of doing the testing of the application using appropriate tools and following various testing methodology. Different tools are available from various vendors with concentration on different type of testing. But the fact also remains that the blending of manual and automated testing methods is the best way to test any application. Testing Automation should concentrate on the following factors: Test process improvements, requirement definition, feasibility, interface testability, maintenance and Reusability.

The Existing system requires a lot of manpower and $\mathrm{UI}$ Test Automation time is more. Silverlight is new Microsoft technology and there is no proper Software for UI Test Automation. This system is developed in such a way that the testers can do UI Test Automation in user - friendly manner.

The Benefits of Software Test Automation Test Automation has a lot of benefits like cost, time and reducing man power etc. Most software development and testing organizations are well aware of the benefits of test automation [12] [13]. A quick glance at the Web sites of any test automation tool vendor will point out a number of the key benefits of test automation. Some of these benefits include:

A. Reduced test execution time and cost: Automated tests take less time to execute than manual tests, and can generally execute unattended. A tester must simply start the test, and then analyze the results when the test is completed [3] [4].

B. Increased test coverage on each testing cycle: Automated tests can allow testing teams to execute large volumes of tests against each build of their application, achieving a level of coverage that would not be possible with manual testing. This increased coverage can help teams uncover bugs in existing functionality much more quickly than through manual testing $[5][6]$. 
C. Increased value of testing: So long as applications are meant for human end users, test automation will never entirely replace the need for human testers. No matter how sophisticated test automation tools become, they will never be as good as human testers at finding bugs in an application.

To one camp it is software testing performed without planning and documentation. The tests are intended to be run only once, unless a defect is discovered. techniques. By freeing manual testers from having to execute repetitive, mundane tests, test automation enables them to focus on using their creativity, knowledge, and instincts to discover important bugs [6] [7].

D. Reduced manual work: Human beings gets tired by doing repeated works more times. But commuters will not tired by doing repeated works more times. User Interface test automation always reduce the manual testing time by automating test process [6] [7].

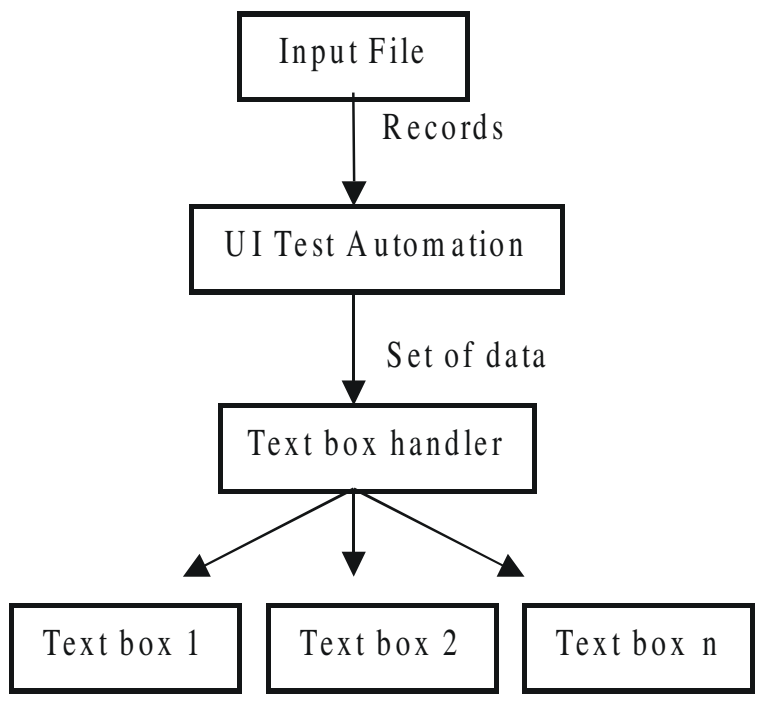

Fig. 1. UI Test Automation Data flow for text box.

\section{Advantages of User Interface Test Automation are:}

* Improved visualization by Micro Soft Dot Net Silverlight

* Online UI Test Automation

* Dot Net Silverlight Applications User Interface Testing

* Testing User Interface with Business logic

* More Secured page User Interface Testing
* Improves the quality of the Applications

- Manual testing is reduced

* Permutation and combinational test cases testing and automation

* Cost and Time is reduced

- Three layers(Ul/Business/Data) testing and automation

* Improves and helps in testing

* Emerging new technology testing and automation

- Accessibility-Testing-Automation-Imputati on

* Checks all possible test cases

* Convert data from XML/Excel To Database Through User Interface

* User Interface Testing and Automation

\section{AGENT TECHNOLOGY}

Agents are autonomous, computational entities that can be viewed as perceiving their environment through sensors and acting upon their environment through effectors. To say that they are autonomous means that to some extent they have control over their behavior and can act without the intervention of humans and other systems.

Intelligent indicates that the agents pursue their goals and execute their tasks such that they optimize some given performance measures. It means that they operate flexibly and rationally in a variety of environmental circumstances. Interacting indicates that the agents may be affected by other agents or possibly by humans in pursuing their goals and executing their tasks. Interaction can take place indirectly through the environment in which they are embedded.

\section{Advantages of Agents in Multi agent systems are:}

* Cooperative agents

* goal oriented

* Decision Support

* Task sharing among agents

* Constrains satisfaction

* Communication and planning among agents

* Coordination and cooperation

The main characteristics of agents in UI Test Automation can be identified as follows: 
Appasami et al : Test Automation for New Generation XAML Web Applications...

* System control is distributed to agents

* Data is decentralized

* Data is partitioned and sent to respective agents

* each agent takes care of their data

* Computation is asynchronous

\section{AGENT BASED UI TEST AUTOMATION FOR SILVERLIGHT APPLICATIONS}

Any work can be easily done by domain experts and particular agents in real life. Similarly our User Interface Test Automation Agents (UITAA) will take care of UI Test Automation of Silverlight Applications. The whole UI Test Automation can be split into several modules based on their functionality. They are UI Accessibility, UI Test, UI Automation and UI Test Automation as shown in figure 2.

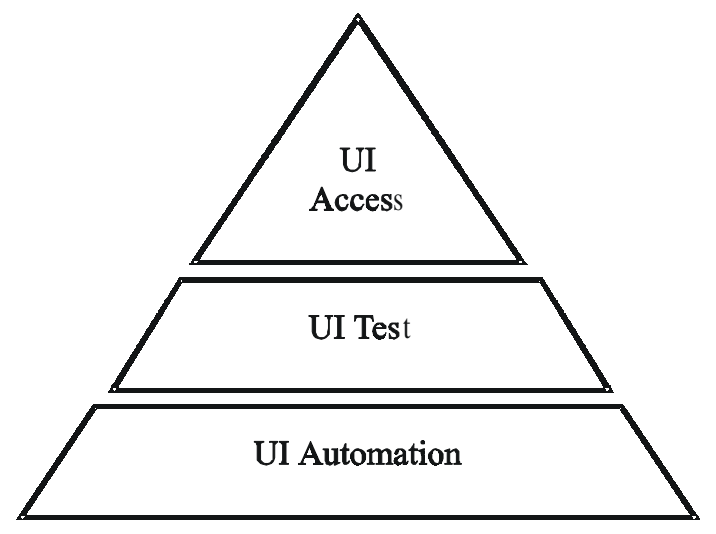

UI Test Automation

Fig. 2. Components of UI Test Automation.

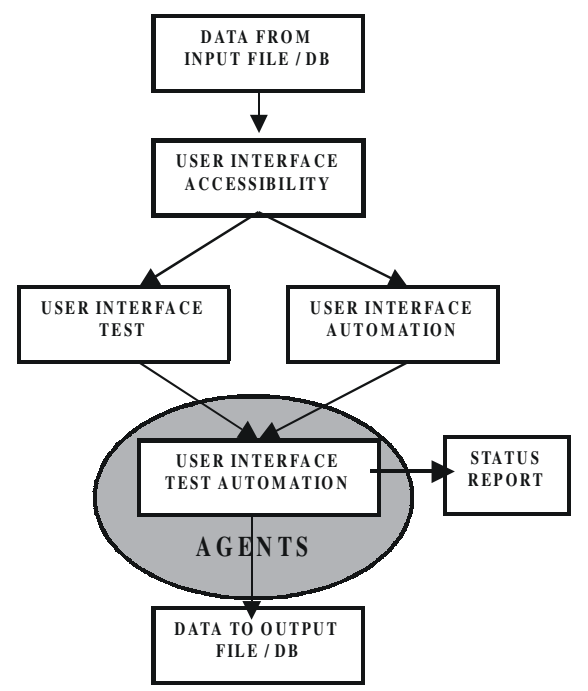

Fig. 3. Agent based UI Test Automation Data flow.
The data flow of the Agent based UI Test Automation is shown in figure3. UI Test and UI Automation are combined to perform UI Test Automation with the help of multi agent system.

\section{IMPLEMENTATION}

Agent based UI Test Automation process for Silverlight applications are implemented as shown in figure4. Our new Agent Based User Interface Test Automation for Silverlight Applications (ABUITASA) gets input record from Excel file. Each record is sent to agents. The agents will split the record and they will send particular data to particular agents to perform UI Test Automation.

The Implementation of Agent Based User Interface Test Automation for Silverlight Applications (ABUITASA) is done in .NET 3.5 framework with C\#.NET, Silverlight, XAML, Excel and Visual studio 2008.

The part of the code for Agent Based User Interface Test Automation for Silverlight Applications is given tin figure5. The textbox_agent is used to pass the values to text boxes and the button_agent is used to perform click events. The supplied records are split into data and distributed to particular agents. The textbox_agent is the responsible agent for all text boxes to set the value for text box. Similarly the button-agent is responsible for button actions.

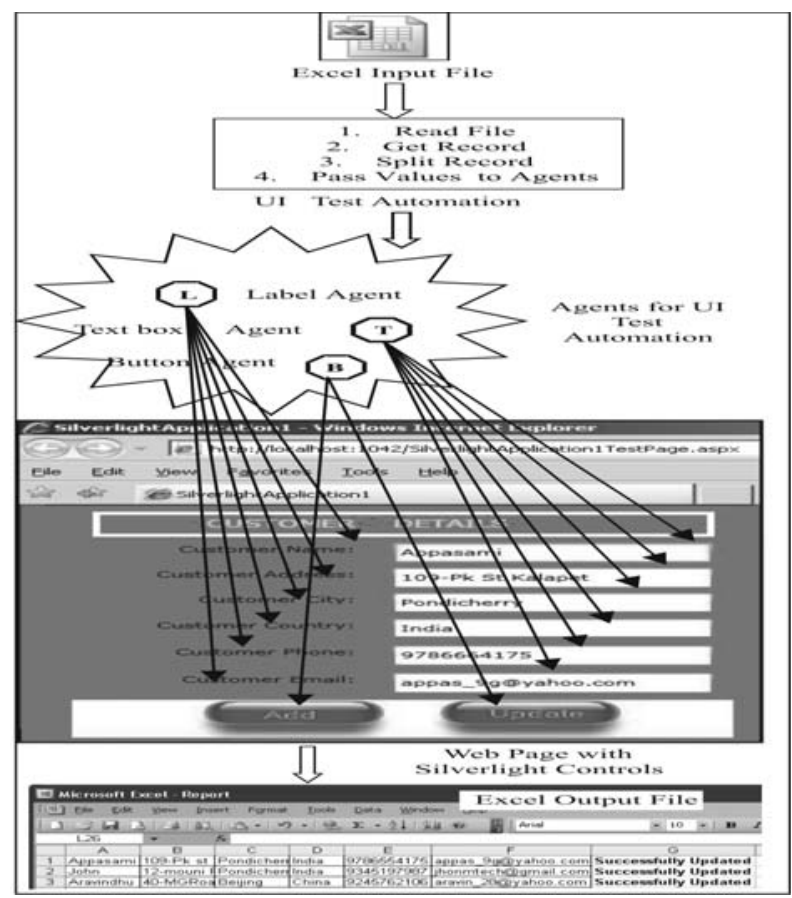

Fig. 4. UI Test Automation Process. 


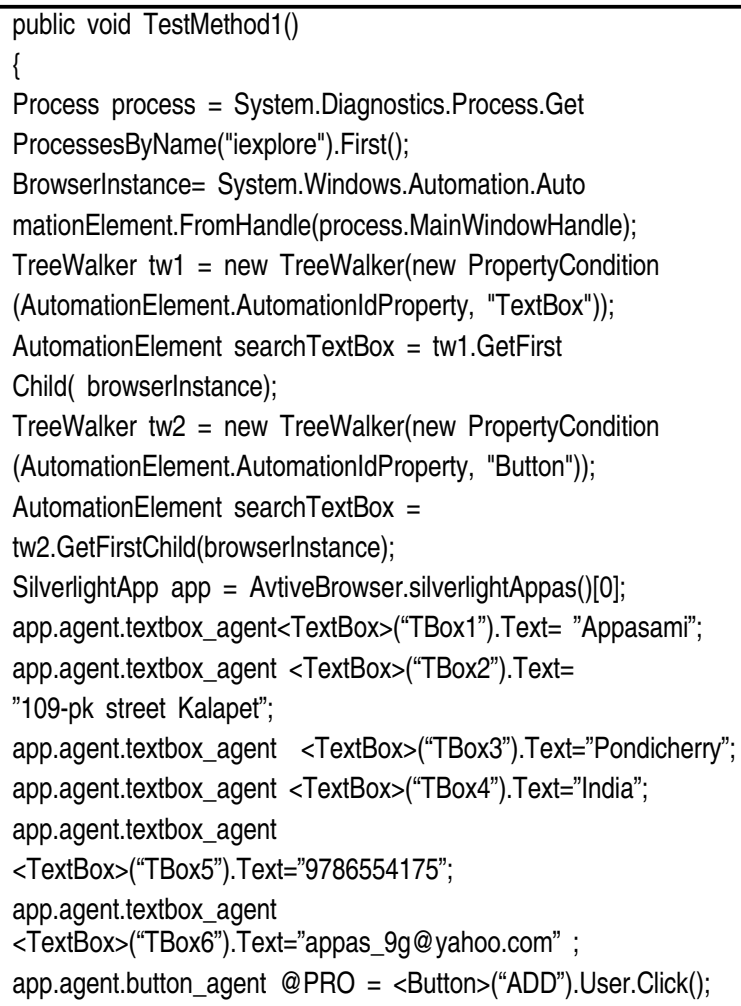

Fig. 5. Sample code for Agent based UI Test Automation of Silverlight application.

Sample code for Agent based UI Test Automation of Silverlight application is given in figure 5 . Initially we have to create one process to get the window handle to control the page. We should create browser handle using window handle to control the window elements. Tree walker entity is created to find a particular element in the web page. Figure5 shows how the values are passed to particular controls through particular agents. For example textbox_agent and button_agent takes care of group of text boxes and buttons.

\section{CONCLUSION}

Agents fill the gap between User Interface Test Automaton and Silverlight applications. Each agent is expert to handle particular type of controls. Agents are simplifies our User Interface Test Automaton. The text box agent is takes care of all text box controls in Silverlight applications to perform User Interface Test Automaton. User Interface Test Automaton plays a vital role in reducing cost, time and man power. It increases the quality of the software.

\section{FUTURE WORK}

In future Multi Agent System User Interface Test Automaton for moonlight applications in Linux environment will be developed. User Interface Test Automaton will reduce man power completely in future.

\section{REFERENCES}

[1] Appasami.G and Suresh Joseph. K, 2009 "User Interface Accessibility and Test Automation for Silverlight Applications", International Journal of Computational Intelligence Research, Research India publications, Vol. 5, No. 2.

[2] Appasami. G and Suresh Joseph. K, Oct 2009 "Performance analysis of various user interface test automation for Silverlight applications", International Journal of Computer and Electrical Engineering, IACSIT, Vol. 1, No. 4 , pages:475-480.

[3] Appasami. G and Suresh Joseph. K, Oct 2009 "Comparative Analysis of Security and Accessibility of Silverlight XAML with Other User Interface Languages", International Journal of Computer Intelligence Research, Research India publications, Vol. 1, No. 4, pages:490-495.

[4] Appasami.G and Suresh Joseph.K, 2009 "Device Independent Visual components using Silverlight", International Engineering and Technology Journal of Advanced Computations, IETECH Publications, ISSN: 0973-8096, Vol. 3, No. 1, pp. 6-13.

[5] Appasami. G and Suresh Joseph. K, 2009 "Automation Peer - User Interface Test Automation for Silverlight Applications", IETECH Journal of Advanced Computations,Vol. 3, No. 2.

[6] Anna Derezinska and Tomasz Malek, June 2007 "Experiences in Testing Automation of a Family of Functional- and GUI-similar Programs", International Journal of Computer Science \& Applications, Technomathematics Research Foundation, Vol. 4, No. 1, pp.13-26.

[7] Zhu Xiaochun, Zhou Bo, Li Juefeng and Gao Qiu, July 2008 "A test automation solution on GUI functional test", IEEE Conference on Software Maintenance , 6(2): pp: 1413-1418.

[8] Xie Q. and Memon A. M., Feb 2007 "Designing and comparing automated test oracles for GUI-based software applications," ACM Transactions on Software Engineering and Methodology, Vol. 16, No. 1, pp. 1-4.

[9] Memon A. M., September 2007 "An event-flow model of GUI-based applications for testing," IEEE conference on Software Testing, Verification and Reliability, Vol. 17, No. 3, pp. 137-157.

[10] Memon A. M., Pollack M. E., and Soffa M. L. Feb. 2001. "Hierarchical GUI test case generation using automated planning". IEEE Transactions on Software Engineering, pages: 144-155, Feb. 2001. 
Appasami et al : Test Automation for New Generation XAML Web Applications...

[11] White L, Almezen H. October 2000 "Generating test cases for GUI responsibilities using complete interaction sequences". Proceedings of the International Symposium on Software Reliability Engineering, 8-11. IEEE Computer Society Press: Piscataway, NJ, 2000; 110-121.

[12] White L, Almezen H, Alzeidi N. November 2001 "User-based testing of GUI sequences and their interaction", Proceedings on Software Reliability Engineering. IEEE Computer Society Press: Piscataway, NJ, 2001; 54-63. 8-11.

[13] Memon A, Nagarajan A, Xie Q. 2005 "Automating regression testing for evolving GUI software". Journal of Software Maintenance and Evolution: Research and Practice; 17(1):27-64.

[14] Memon A. M. and Xie. Q. 2005 "Studying the fault-detection e.ectiveness of GUI test cases for rapidly evolving software". IEEE Transactions on Software Engineering, 31(10):884-896.

[15] Xie Q. and Memon A. M. 2007 "Designing and comparing automated test oracles for GUl-based software Applications". ACM Trans on Software Engineering and Methodology, 16(1):4.

[16] Appasami.G and Suresh Joseph. K, June 2009 "Automation Peer - User Interface Test Automation for Silverlight Applications", IETECH Journal of Advanced Computations, India.

[17] Sycara K. P. and Wollridge M., 1998 "Proceedings of the Second International Conference on Autonomous agents", Association for Computing Machinary, Inc. (ACM).

[18] Demazeau Y., 1998 "Proceedings of the third International Conference on Multi-Agent Systems", ICMAS-98, IEEE Computer Society.

[19] Gerhard Wheiss, 1999 "Multiagent Systems", the MIT Press.

[20] Fewster, 1999 "Software Test Automation", Addison Wesley.

[21] Kanglin Li and Mengqi Wu, 2005 "Effective GUI Test Automation: Developing an Automated GUI Testing Tool ", SYBEX Inc.

[22] Kanglin Li and Menqi Wu, 2004 "Effective Software Test Automation: Developing an Automated Software Testing Tool" ISBN:0782143202 Sybex Inc.
[23] Tom Arnold, Dominic Hopton, Andy Leonard and Mike Frost, 2007 "Professional Software Testing with Visual Studio® 2005 Team System", Wiley Publishing, Inc.

[24] Elfriede Dustin, 2003 "Effective Software testing", Pearson Education Inc.

[25] Brad Dayley and Lisa DaNae Dayley, 2008 "Silverlight" 2 Bible", Wiley Publishing, Inc., 2008.

[26] Matthew MacDonald, "Silverlight 2 Visual Essentials" Firstpress.

[27] http://www.silverlight.net

[28] http://code.msdn.microsoft.com/silverlightut

[29] http://silverlight.net/learn/tutorials/controls.aspx

[30] http://www.jeff.wilcox.name/2008/03/silverlight2-unit-tes ting

[31] http://msdn.microsoft.com/en-us/library/ cc645045(VS.95).aspx

[32] http://weblogs.asp.net/scottgu/archive/2008 /04/02/unit-testing-with-silverlight.aspx

[33] http://dotnetslackers.com/Patterns_and_Practices/UI_ Automation_Testing_with_UIA_Veify.aspx

[34] http://publib.boulder.ibm.com/iseries/v5r1/ic2924/index. htm?info/rzakl/rzaklintroadvantages.htm

[35] http://artoftestinc.blogspot.com/2008/08/automated-test ing-of-silverlight.html

[36] http://it.toolbox.com/wiki/index.php/Oracle_11G_New_ Features

[37] http://www.adp-gmbh.ch/ora/misc/features.html

[38] http://www.codeguru.com/forum/archive/index.php/t-20 4260.html

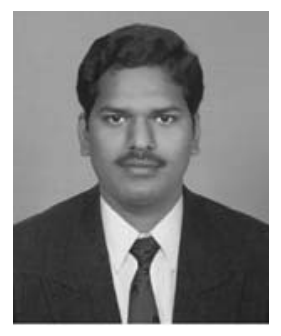

Mr. G. Appasami received his Master of Science degree in Mathematics, Master of Computer Applications and Master of Technology in Computer Science and Engineering from Pondicherry University, Pondicherry, India.

Currently he is working as Assistant Professor in the Department of Computer Science and Engineering, Dr. Pauls Engineering College, Villupuram, Tamil Nadu, India, afiliated to Anna University Chennai. His Area of interests includes image processing and web technology. 